\author{
SCANNING TRANSIENT CURRENT STUDY OF THE I-V STABILIZATION \\ PHENOMENA IN SIUICON DETECTORS IRRADIATED BY FAST NEUTRONS*
}

\author{
V. Eremin and E. Verbitskaya \\ Ioffe Physico-Technical Institute, Russian Academy of Sciences \\ 194021 St. Petersburg, Russia
}

Z. Li

Brookhaven National Laboratory

Upton, NY 11973

\begin{abstract}
A. Sidorov
Russian Institute of Material Science and Technology

Moscow, Zelenograd, Russia
\end{abstract}

E. Fretwurst and G. Lindstroem

I. Institut fur Experimentalphysik, Universitat Hamburg

Hamburg, Germany

March, 1996

*This research was supported in part by the U. S. Department of Energy:

Contract No. DE-AC02-76CH00016. 


\section{DISCLAMMBR}

Portions of this doesment may be illegible in electronic inage products. Inages are produced from the best avnilable original document. 


\title{
Scanning Transient Current study of the $I-V$ stabilization phenomena in silicon detectors irradiated by fast neutrons
}

\author{
V. Eremin, and E. Verbitskaya \\ Ioffe Physico-Technical Institute, Russian Academy of Sciences, \\ 194021 St-Petersburg, Russia \\ Z. $\mathrm{Li}$ \\ Brookhaven National Laboratory, \\ Upton, New York 11973, USA \\ A. Sidorov \\ Russian Institute of Material Science and Technology \\ Moscow, Zelenograd, Russia \\ E. Fretwurst, and G. Lindstrom, \\ I.Institut fur Experimentalphysik, Universitat Hamburg, \\ Hamburg, Germany
}

\begin{abstract}
Investigation of the $I-V$ stabilization phenomena in neutron irradiated silicon detectors has been carried out using scanning transient current technique (STCT) on non-irradiated $\mathrm{p}^{+}-\mathrm{p}-\mathrm{n}^{+}$detectors. The $\mathrm{p}^{+}-$ $\mathrm{p}-\mathrm{n}^{+}$detectors were used to simulate the $\mathrm{p}^{+}-\mathrm{n}-\mathrm{n}^{+}$detectors irradiated beyond the space charge sign inversion (SCSI). Two mechanisms partially responsible for the $I-V$ stabilization have been identified.

\section{INTRODUCTION}

This work presents experimental results on the investigation of the electric field distribution near the surface of Si planar detectors fabricated from p-type silicon. It was stimulated by the experimental fact that junctions of $\mathrm{p}^{+}-\mathrm{n}-\mathrm{n}^{+}$detectors made from high resistivity silicon switch from near the $\mathrm{p}^{+}$side to near the $\mathrm{n}^{+}$side (termed as space charge sign inversion (SCSI) or "inversion") after neutron radiation with fluences $>10^{13} \mathrm{~cm}^{-2}$ without any significant, sudden changes in their current-voltage characteristics [1-2].

Usually the construction of Si planar detectors contains the elements for minimization of the leakage current and low voltage breakdown like overlapping metallization with silicon dioxide layer, guard rings and passivation of the area between the main $\mathrm{p}^{+}-\mathrm{n}$ junction and the border of the detector chip. This approach for $I-V$ stabilisation does actually work on the side of $\mathrm{p}-\mathrm{n}$ junction where the electric field achieves its maximum, i.e. on the $\mathrm{p}^{+}$-side for detectors processed from $\mathrm{n}$-type $\mathrm{Si}$. Irradiation of such detectors by fast neutrons leads to the inversion of the space charge sign in the space charge region (SCR) so the junction changes their position and becomes located at the $\mathrm{n}^{+}$-side. Consequently the electric field maximum moves to the $\mathrm{n}^{+}$-contact which has not any elements to hinder the broadening of the electric field region till the borders of the detector chip. As the border is very damaged by scribing, a significant increase of the leakage current should occur due to surface leakage and/or high field breakdown near the edges.
\end{abstract}


In reality, however, measurements on irradiated $\mathrm{p}^{+}-\mathrm{n}-\mathrm{n}^{+}$detectors have not shown significant leakage current increase near the SCSI point and these detectors may be operated up to 1000 volts without apparent breakdown. One of the possible mechanisms of the $I-V$ stabilisation based on the deep level trapping effect was proposed early in ref. [3]. The idea is that defects, generated in Si bulk by neutron irradiation, capture the carriers injected from the damaged detector border and accumulate an additional space charge in the opposite sign which suppresses the electric field near the points of injection. This process works like a negative feed back that stabilizes the carrier injection and therefore the detector leakage current. In this paper, other possible mechanisms that may affect the $I-V$ stabilization are investigated in terms of the electric field and potential distribution over the detector chip surface. Scanning transient current technique (STCT) is used in the study.

\section{SAMPLES AND EXPERIMENTAL TECHNIQUE}

To eliminate the leakage current effects related to the current generated in the bulk of the detectors, samples with $\mathrm{p}^{+}-\mathrm{p}-\mathrm{n}^{+}$configuration fabricated in BNL from $5 \mathrm{k} \Omega$-cm $\mathrm{p}$-type silicon have been used as models of the irradiated, inverted $\mathrm{p}^{+}-\mathrm{n}-\mathrm{n}^{+}$detectors. The detector area was $4 \times 4 \mathrm{~mm}^{2}$ with a thickness of $350 \mu \mathrm{m}$. The area of chip was $6 \times 6 \mathrm{~mm}^{2}$. On the $\mathrm{p}^{+}$side, the detector construction includes the $\mathrm{p}^{+}$contact (boron implantation) covered by $\mathrm{Al}, \mathrm{a} \mathrm{p}^{+}$guard ring, and a $\mathrm{SiO}_{2}$ passivation area between the $\mathrm{p}^{+}$contact and the $\mathrm{p}^{+}$guard ring and periphery of the chip. The $\mathrm{n}^{+}$contact was made by phosphorus implantation over the entire back side of the wafer and was not protected in any way. On both sides of a detector, optical windows were made in $\mathrm{Al}$ metallization to allow the generation of electron hole pairs in silicon bulk by a laser light.

The TCT set-up for the investigation of the electric field distribution has been described earlier in ref.[4]. A laser diode with wave length of $834 \mathrm{~nm}$ and light pulse duration less than $0.8 \mathrm{~ns}$ was used. The penetration depth of the light in $\mathrm{Si}$ is about $20 \mu \mathrm{m}$. For laser scanning measurements, the cut end of an optical fiber that was used as a collimator was arranged on a X-Y movable table to control the position of the light spot on the detector surface. The lateral resolution of the system is determined by the diameter of light spot which is equal to the diameter of optical fiber collimator of about $50 \mu \mathrm{m}$.

\section{EXPERIMENTAL RESULTS}

The as processed $\mathrm{p}^{+}-\mathrm{p}-\mathrm{n}^{+}$detector prototypes have been characterized by $I-V$ measurements (Fig. 1a). The leakage current curve 1 in Fig. 1a was obtained with the $\mathrm{p}^{+}$guard-ring floating (GRF, as shown in Fig. 1b) while the leakage current curve 2 was obtained with detector $\mathrm{p}^{+}$-contact and the $\mathrm{p}^{+}$guard-ring shorted together (guard-ring connected, GRC, as shown in Fig. 1b). Also shown in Fig. 1a is the potential of the floating $\mathrm{p}^{+}$guard-ring corresponding to leakage current curve 1 (GRF). As the leakage current was rather large, the depletion voltage was determined by TCT instead of the traditional $C-V$ technique [4]. Fig. 2a shows the hole current response (laser illumination on the $\mathrm{n}^{+}$contact) at various bias. The initial peaks in the current shapes are caused by small contribution of electrons. The hole currents go down with time, indicating that the high field (or the junction) is on the $\mathrm{n}^{+}$side. Fig. $2 \mathrm{~b}$ shows the electron current response (laser illumination on the $\mathrm{p}^{+}$contact) at various bias. The drop-off of the hole current response exponential decay (Fig. 2b) and the elimination of the electron current response tail (Fig. 2a) indicate the pinch-off at the depletion voltage $V_{d}=300 \mathrm{~V}$. As shown in Fig. 3a, the current pulse shapes measured by scanning of the periphery between the $\mathrm{p}^{+}$and $\mathrm{n}^{+}$contacts (the light beam is aimed to the detector cross section between 
the contacts) show significnt signal decreases (about 100 times smaller) as compared to the normal ones. The low sensitivity of the detector border region has been seen affected little by the detector bias in a wide range of the bias (Fig. 3b).

\section{MODEL AND DISCUSSION}

The model of the $I-V$ stabilization for a $\mathrm{p}^{+}-\mathrm{p}-\mathrm{n}^{+}$detector that explains the main peculiarities of the electric field distribution is shown in Fig.4. As the detectors studied here was not irradiated, detector bulk generation current can be neglected. The carriers generated on the scribed surface and in the layer nearby with the large density of the defects can reach the SCR of the detector either by drifting or diffusion. In this case these carriers make up the main contribution to the detector leakage current. Therefore the leakage current must follow the depth of the SCR and increases with bias (since the area of surface generation increases with the SCR depth), which is confirmed by data shown in by Fig.1 (curve 2). After monotonical increase the current becomes essentially stable starting from the bias enough for full depletion $\left(V_{d}=300\right.$ V). At this bias the potential of the $\mathrm{p}^{+}$guard-ring (which is floating) begins to increase with bias (Fig.1). In other words, the potential applied to the detector periphery increases much slowly than in the central part of the detector as part of the bias drops between the $\mathrm{p}^{+}$contact and the guard ring. Fig. $4 \mathrm{~b}$ shows the detector cross section in the area with $\mathrm{p}^{+}$contact and $\mathrm{p}^{+}$guard ring. The positive charge in $\mathrm{SiO}_{2}$ creates a inverted $\mathrm{n}^{+}$-type layer under the oxide. The voltage drop applied to the region [a,b], defined as the "gap" , biases the junction originated from the $\mathrm{p}^{+}$guard ring to the inverted $\mathrm{n}^{+}$layer (near point $\mathrm{b}$ ) in the forward direction. The second junction between the $\mathrm{p}^{+}$contact and the inverted $\mathrm{n}^{+}$layer (near point a), on the other hand, is biased in the reverse direction, which prevents leakage current to flow in the inverted layer under the surface. The arrows show the possible path of leakage current (surface generated/injected hole current) in the $p$ region and near the $\mathrm{p}^{+}$region of the detector.

The structure in Fig. $4 \mathrm{~b}$ can be considered as that of a field effect transistor with p-channel and a gate $\left(\mathrm{n}^{+}\right.$-inverted layer), the latter is in contact with a source $\left(\mathrm{p}^{+}\right.$guard ring). The potential drop between the $\mathrm{p}^{+}$guard ring and the $\mathrm{p}^{+}$contact is determined by the conductivity of the $\mathrm{p}^{+}-\mathrm{n}$ junction at point a and by the bias between the $\mathrm{n}^{+}$layer and the $\mathrm{p}^{+}$contact. Measurements on other (but similar) $\mathrm{p}^{+}-\mathrm{p}-\mathrm{n}^{+}$detectors show that the bias drop $V_{r}$ in this region is a significant part (up to 60\%) of the over depletion voltage $V_{o d}$ $=V-V_{d}$.

To understand the role of the gap in $I-V$ stabilization, two schemes of current measurement were used (Fig.1, b). The first scheme corresponds to the standard measurements of $\mathrm{p}^{+}$guard ring potential and the detector leakage current. In this case there are two mechanism that may restrict the leakage current: the resistivity of the gap and the finite value of the damaged-edge (scribed-border) generated current (surface current). In the second scheme the gap is shorted and the leakage current can only be restricted by the current generated at the damaged-edge current (surface current). The results of these measurements are illustrated in Fig.1, with curve 1 for scheme 1 and curve 2 for scheme 2, respectively. It is clear that no significant difference can be found between the $I-V$ characteristics measured with the two different schemes. Therefore the resistivity of the gap as a mechanism of the leakage current restriction can be ruled out and the main mechanism of the observed $I-V$ stabilization is a limited generation of the carriers at the scribed border of the detector chip. The gap effect could, however, significantly improve the $I-V$ characteristic at high biases near the breakdown voltage when injection of the carriers into the SCR can be related to the impact ionization in the areas with the electric field close to that causing breakdown. This is 
because that gap effect does reduce the over-all potential difference between the $\mathrm{p}^{+}$guard ring and the point $\mathrm{c}$ on the $\mathrm{n}^{+}$contact (Fig. $4 \mathrm{~b}$ ), which results in lower field near point $\mathrm{c}$.

To estimate the value of the leakage current caused by damaged-edge, a third measurement scheme has been used. In this scheme, the $\mathrm{p}^{+}$guard ring was grounded (GRG, see Fig. 1b)) so that current flowing through the electrode of the $\mathrm{p}^{+}$guard ring does not contribute to the detector leakage measurement, which was measured through the $\mathrm{p}^{+}$-contact. Fig. 5 shows such measured $I-V$ characteristic of the same $\mathrm{p}^{+}-\mathrm{p}-\mathrm{n}^{+}$ detector as shown in Fig. 1. It is clear that at or near full depletion voltage the leakage current drops down to the value corresponding to the bulk generation current (about $0.2 \mu \mathrm{A}$ ). The surface current is now flowing through the $\mathrm{p}^{+}$guard ring (pinch-off effect). This $I-V$ characteristic is in total agreement with proposed model: the leakage current is mainly the current generated in the damaged layer under the scribed surface (about $7 \mu \mathrm{A}$ ). The value of the surface generation component depends on the electric field distribution near the surface and the degree of the damage on the surface. Comparison of the current response (TCT) measured on the contacts (Fig.2) with that at the chip border (Fig. 3) shows much lower value of collection efficiency of charges generated near the border. One possible explanation is that there exists a layer near the border within which there is little or no electric field (Fig. 4). The depth of this nofield layer, or "dead layer", can be comparable to the penetration depth of the laser light that is about 20 $\mu \mathrm{m}$ for the laser wavelength of $830 \mathrm{~nm}$ used in this study.

\section{CONCLUSION}

The investigation carried out in this work have identified two additional mechanisms that may affect the $I-V$ stabilization of neutron irradiated silicon detectors: the channel with high resistance between $\mathrm{p}+-$ implants and the limited extraction of the carriers from the damaged border of detector chip. Fig. 6 presents the $I-V$ curve for a planar $\mathrm{p}^{+}-\mathrm{p}-\mathrm{n}^{+}$detector irradiated by neutrons to a fluence of $7.6 \times 10^{12} \mathrm{n} / \mathrm{cm}^{2}$. The same peculiarity exists - stabilization of the current for biases higher than the depletion voltage. Note that the $I-V$ characteristic is smoother as compared to that of a non-irradiated $\mathrm{p}^{+}-\mathrm{p}-\mathrm{n}^{+}$detector (Fig.1) that could be affected by irradiation induced defects with deep levels on the current injection from the surface described earlier in ref. [3].

\section{Acknowledgements}

The authors would like to thank Dr. V. Kozlov for the discussion and I. Iliashenko for the help in the measurements. 


\section{References}

1. E. Fretwurst et al., Nucl Instr. Meth., A326, 326(1994).

2. Z. Li et al., IEEE Trans. Nucl. Sci. NS-42, No. 4, August (1995) 219

3. V. Eremin, Z. Li, to be published in Nucl. Instr. Meth.

4. V. Eremin et al.. to be published in Nucl. Instr. Meth. A, 1996 (in press). 
Figure Captions

Fig. 1. Current-voltage characteristics for detector \# 16-79: curve 1 is measured with scheme 1 (GRF)shown in Fig. 1b, curve 2 is measured with scheme 2 (GRC) shown in Fig. 1b. Solid thick curve show the potential of the $\mathrm{p}^{+}$-guard ring measured with scheme 1 .

Fig. 2. Current response curves for detector \# 16-79 measured by TCT to determine the full depletion voltage: a) laser pulse on the $\mathrm{n}^{+}$-contact, b) laser pulse on the $\mathrm{p}^{+}$-contact.

Fig. 3. STCT data of detector \#16-79 (the curves were shifted along the vertical direction for clear comparison): a) current response curves measured by scanning of the chip periphery. The detector was biased at 370 volts and the scanning step is $100 \mu \mathrm{m}$. b) current response curves measured at different bias. Laser spot position is of $75 \mu \mathrm{m}$ from the $\mathrm{n}^{+}$-contact .

Fig. 4. Model of the electric field distribution in a $\mathrm{p}^{+}-\mathrm{p}-\mathrm{n}^{+}$detector: a) detector biased at $V<V_{f d}$, and b) detector biased at $V>V_{f d}$. Dashed line is the contour of the space charge region (SCR). The horizontal arrows in a) indicate injection of charge carriers generated near the scribed border of the chip.

Fig. 5 Current voltage characteristics of a $\mathrm{p}^{+}-\mathrm{p}-\mathrm{n}^{+}$detector measured with $\mathrm{p}^{+}$-guard ring grounded (GRG).

Fig. 6. Current voltage characteristics of $a p^{+}-p-n^{+}$detector irradiated by fast neutrons to a fluence of $7.6 \times 10^{12} \mathrm{n} / \mathrm{cm}^{2}$. 


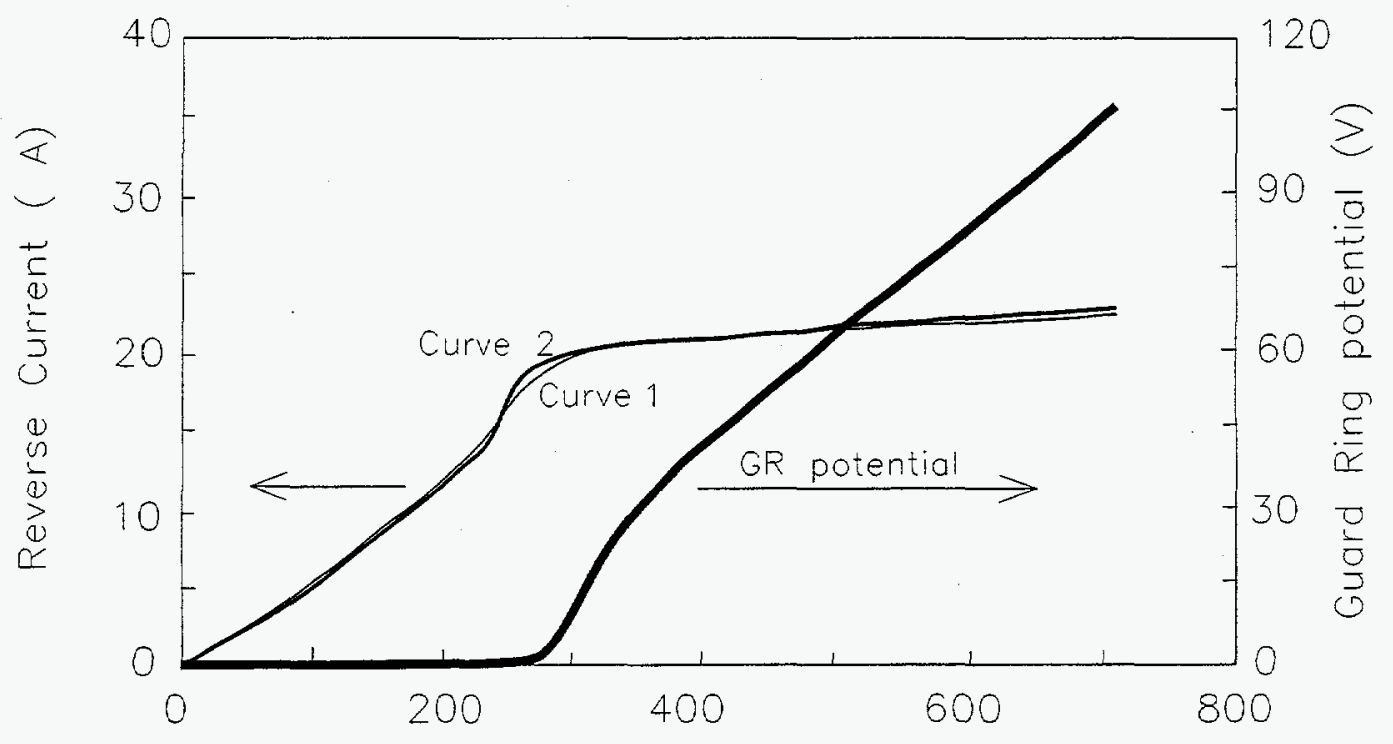

a)
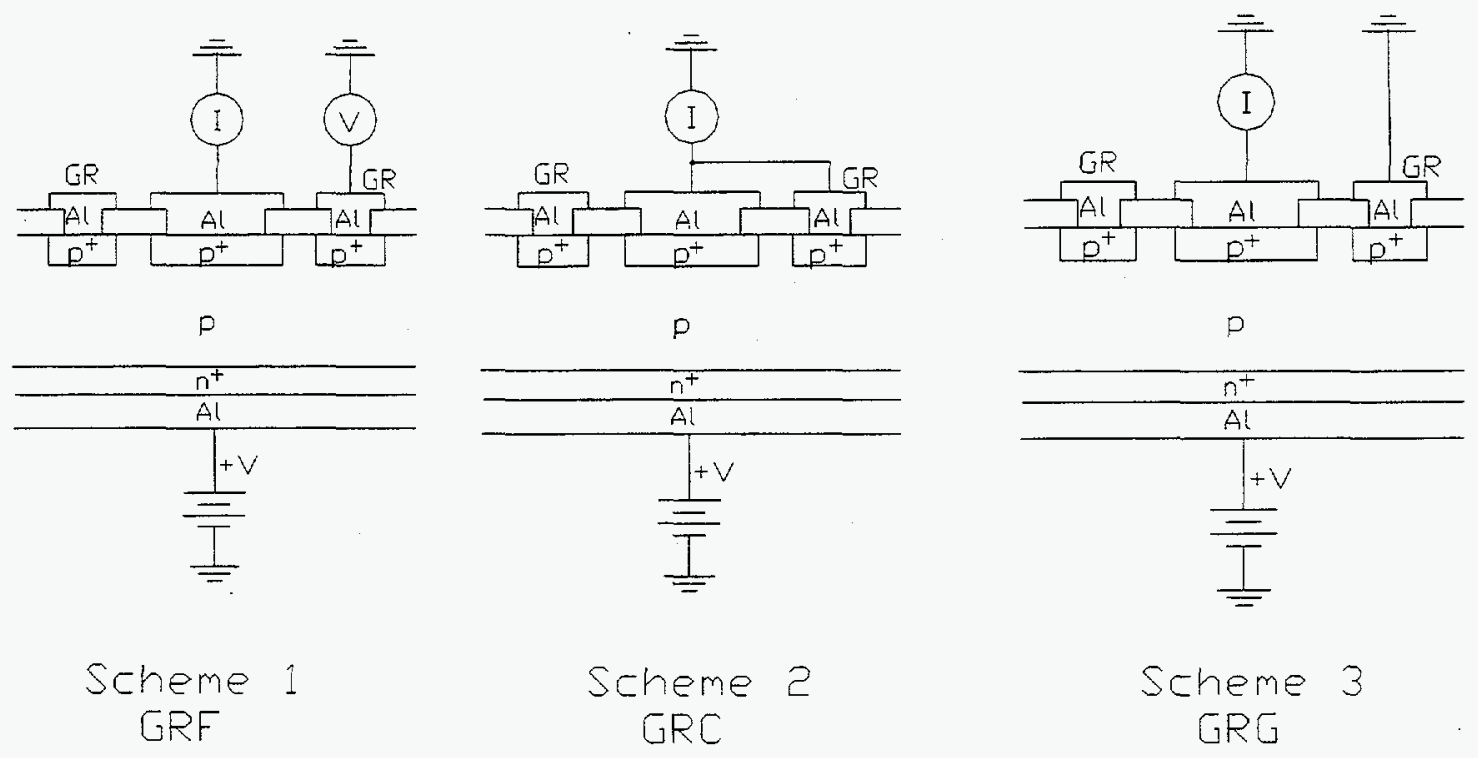

Scheme 2
GRC

Scheme 3 GRG

b) 

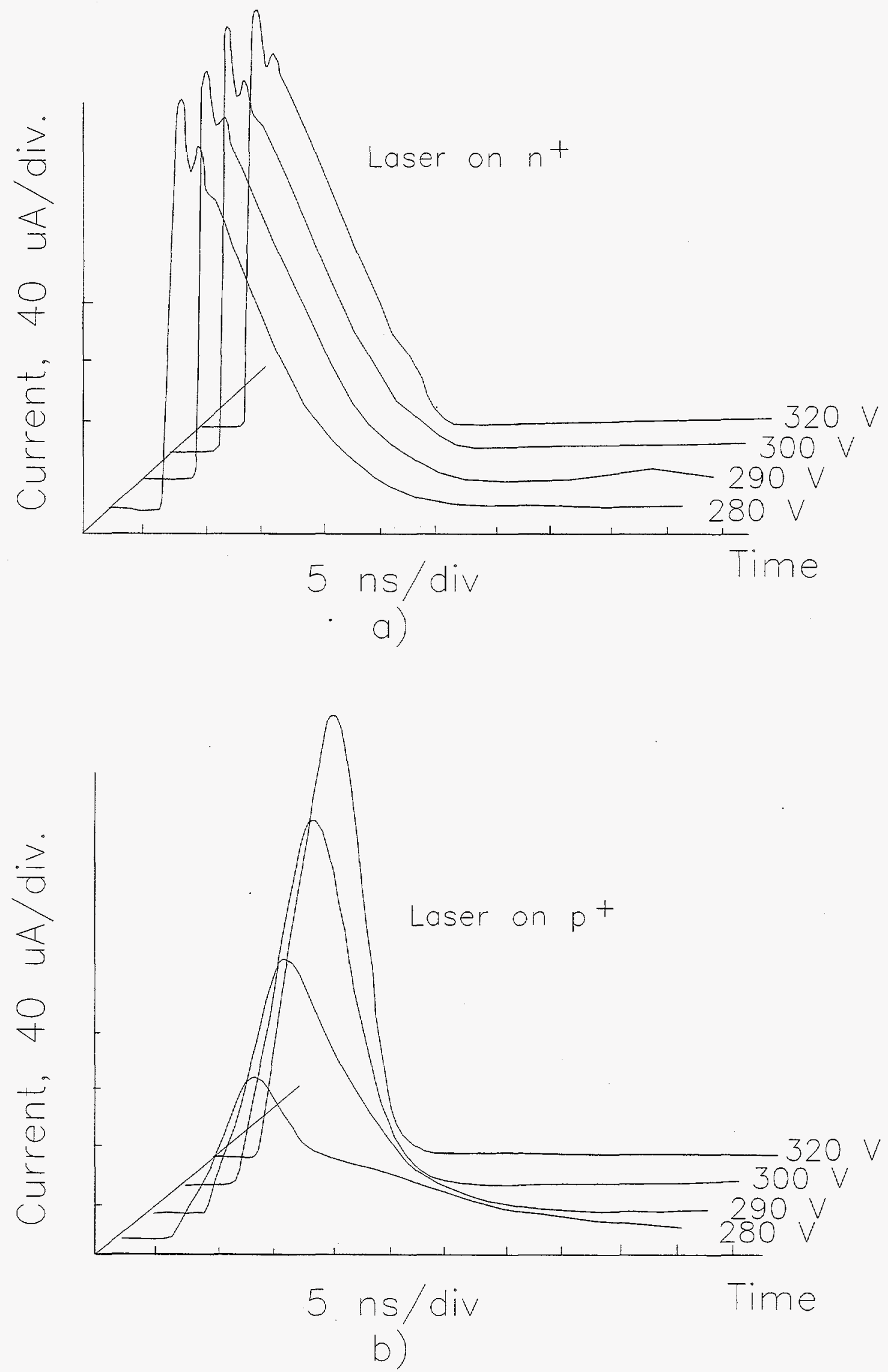

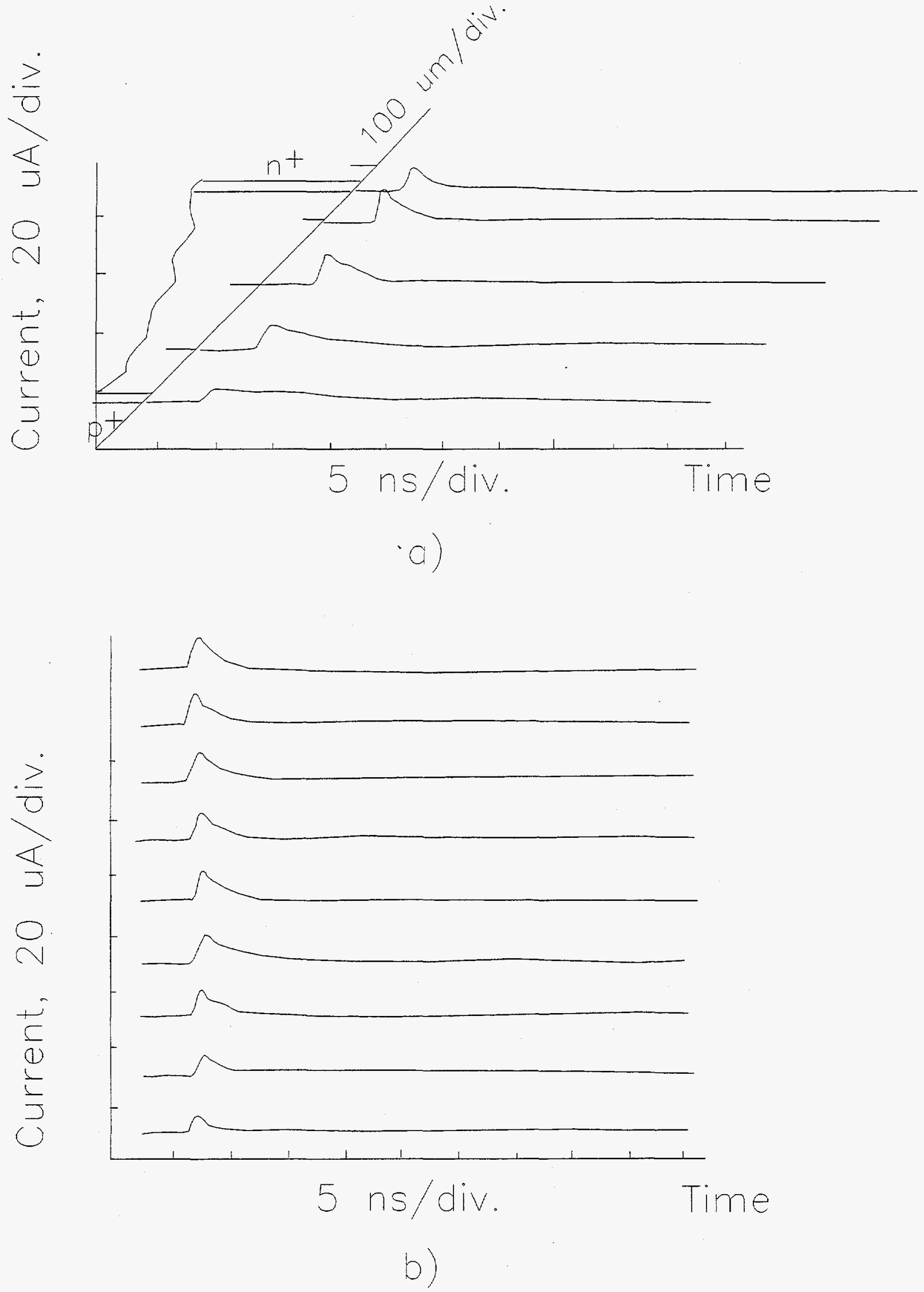


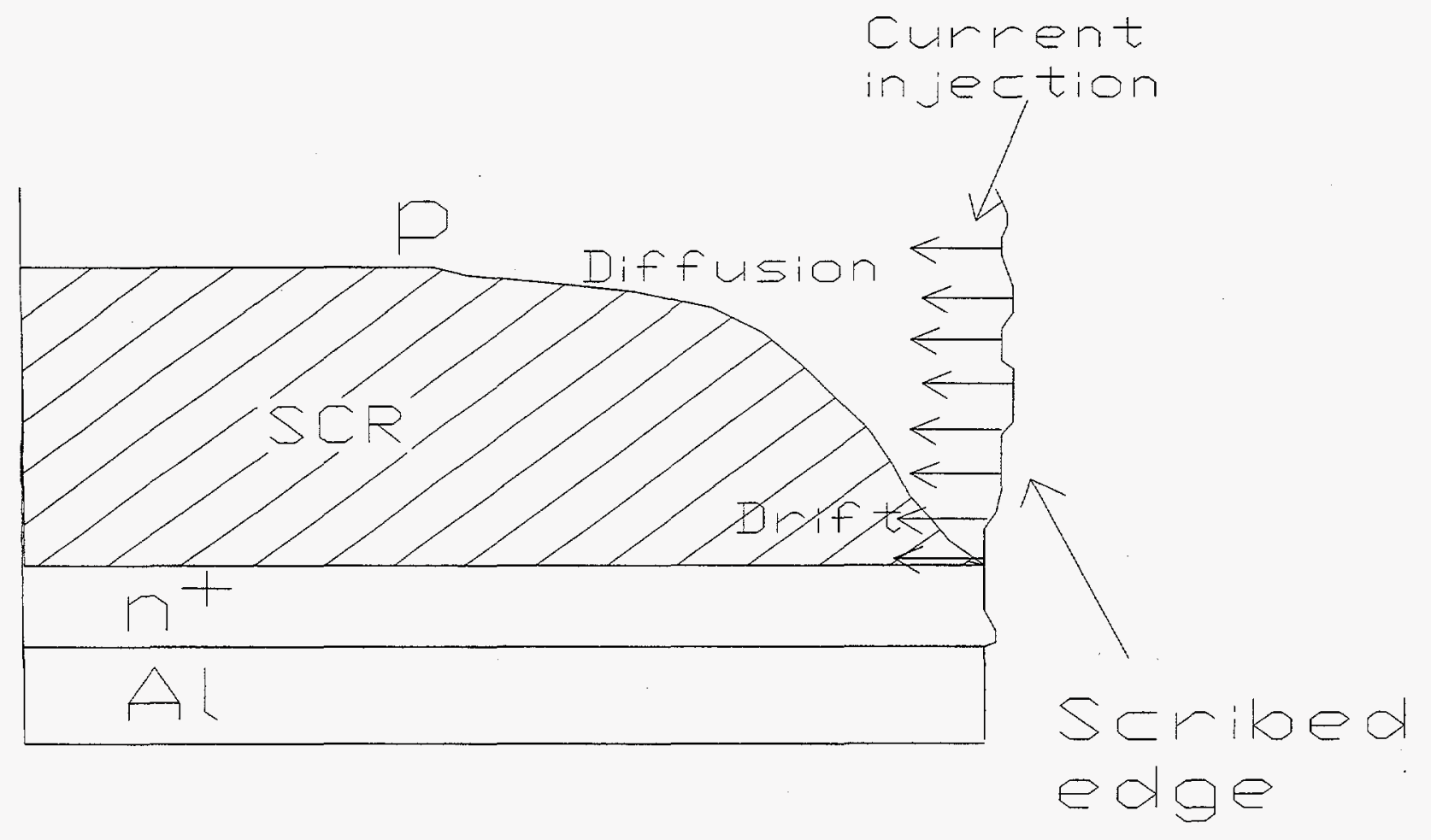

a)

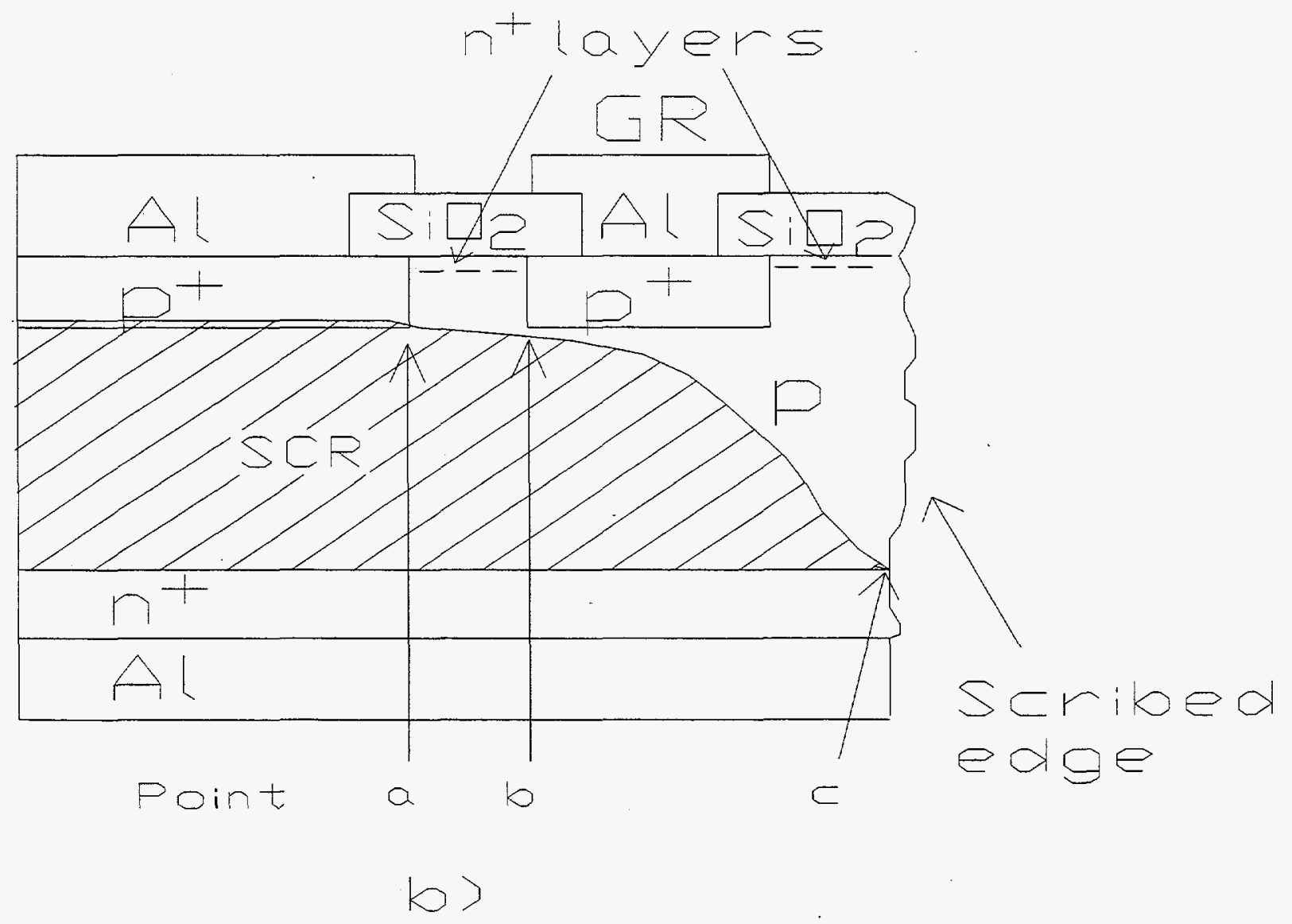




\section{I-V Characteristics}

\#79, p+/p/n+ diode

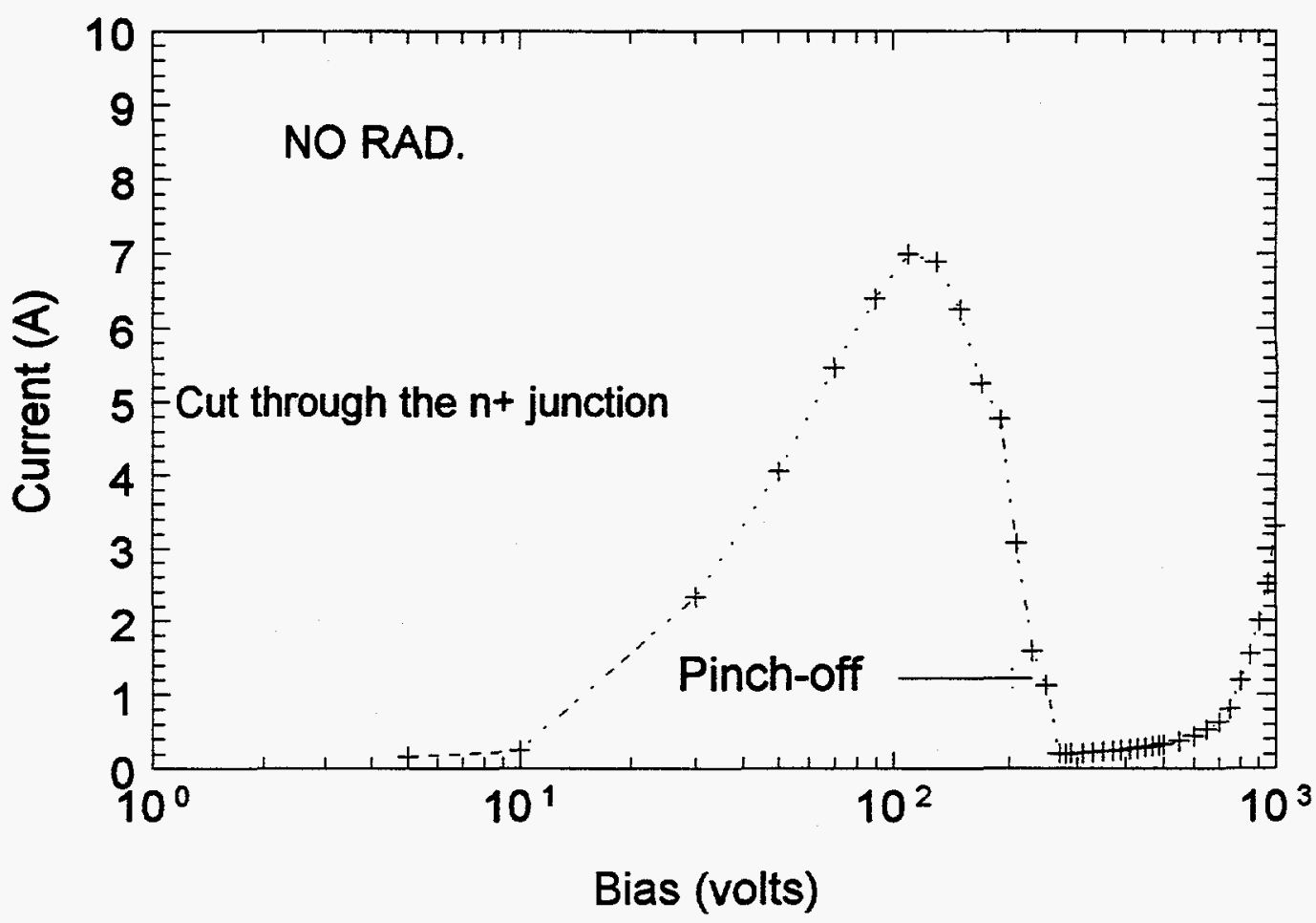

P+P: Fig5.TC 


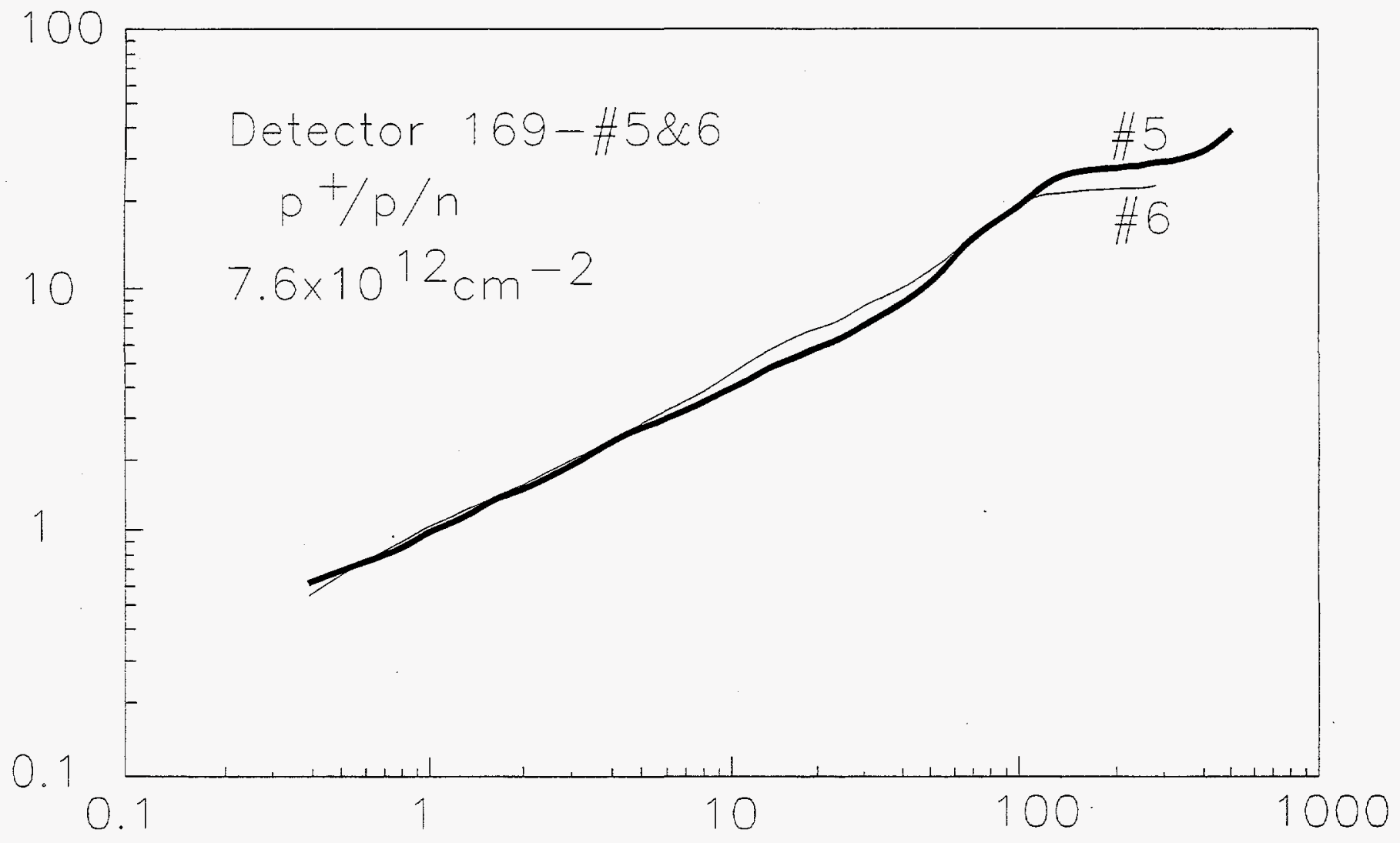

